\title{
Application of platelet-rich plasma in the treatment of chronic skin ulcer - Case report
}

\author{
Jane Marcy Neffá Pinto ${ }^{1}$ \\ Hye Chung Kang ${ }^{1}$
}

\author{
Natássia Soares Pizani ${ }^{1}$ \\ Luis Augusto Knecht Silva ${ }^{1}$
}

\section{DOI: http://dx.doi.org/10.1590/abd1806-4841.20143004}

Abstract: The platelet-rich plasma (PRP) has proved promising regarding its applicability in dermatology, especially in the healing of chronic ulcers. The autologous platelet-rich plasma is obtained by centrifuging the blood, so that the components are separated by density gradient. The final product is a gel rich in growth factors that act in tissue repair by activating fibroblasts and inducing extracellular matrix remodeling.

Keywords: Platelet-rich plasma; Skin ulcer; Wound healing

\section{INTRODUCTION}

Cutaneous ulceration is a very common event, with current prevalence around $0.18 \%$ and $0.32 \%$, incidence of $0.78 \%$ and a clear tendency of rising with the increasing mean age of the population. The effects on socioeconomic costs are inevitable. Chronic cutaneous ulcers have a great impact on patient quality of life and public health costs in Brazil and worldwide. The European Union reserves $2 \%$ of its yearly health budget for the treatment of such ailments. ${ }^{1}$

Healing of wounds is a complex process mediated by signals of molecular interaction involving recruiting of mesenchymal cells, proliferation and regeneration of the extracellular matrix. The healing process is a response of innate immunity for the restoration of tissue integrity. It is regulated by a pattern of events including coagulation, inflammation, granulation tissue formation, epithelialization and tissue remodeling. These events are mediated by cytokines and growth factors that modulate such cellular activities.

\section{CASE REPORT}

S.M.V.S., 65-year-old, female, black, retired teacher, presents cutaneous ulcers with 13 years of evolution in the medial malleolar region of right lower limb and middle third of left lower limb (Figure 1). The lesions arise from chronic venous insufficiency. Initially, they measured around $2 \mathrm{~cm}$. However, there was progressive increase of lesion diameter since their onset. Between the period from 2007 to 2009, she used curative dressings based on essential fatty acids (EFA) every 15 days. Around 5 years ago, she started treatment with hyperbaric oxygen therapy, 80 doses divided into 3 sessions, with broad and irregular intervals, with no apparent improvement. The patient underwent lesion biopsy which showed dense vascularized connective tissue exhibiting fibroplasia, acute and chronic inflammatory infiltrate close to the granulation tissue and ulcer material. From November 2012 onward she started taking regular doses of PRP on the left lower limb lesion. Currently, a painful ulcer remains presenting granulation tissue with irregular edges, discrete fibrinous exudate and adjacent hyperchromia. Reepithelialization areas in the interior of the lesion were observed (Figures 2 and 3). Absence of phlogistic signs. Currently using Diosmin ${ }^{\circledR}$, Vasogard ${ }^{\circledR}$, Dermavite ${ }^{\circledR}$, Drenison ${ }^{\circledR}$ cream.

Obese patient, not diabetic and hypertensive undergoing regular treatment. She denied smoking or alcohol consumption.

Since the beginning of PRP regular administration there was noticeable improvement of the lesion, observed by the increase of granulation tissue. Softening of borders can be noticed by palpation, which may be interpreted as decrease of local fibrosis. Currently, she presents skin patches in the interior of the ulcer, corresponding to reepithelialization (Figure 3).

Approved by the Advisory Board and accepted for publication on 20.08.2013

* Work performed at Hospital Universitário Antônio Pedro - Universidade Federal Fluminense (HUAP-UFF) - Niterói (RJ), Brazil.
Financial Support: none

Conflict of Interests: none

Universidade Federal Fluminense (UFF) - Niterói (RJ), Brazil. 


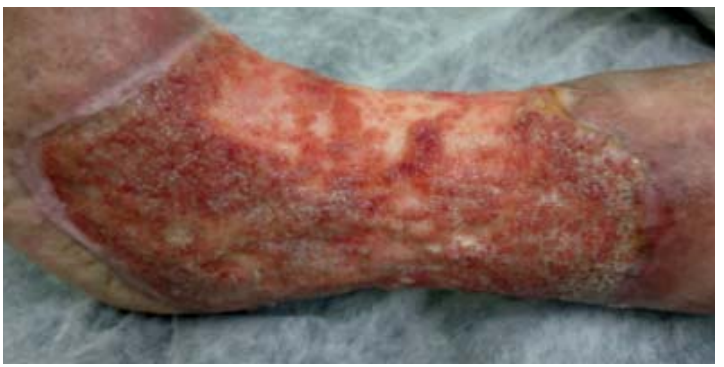

Figure 1: Lower left limb - June/2011

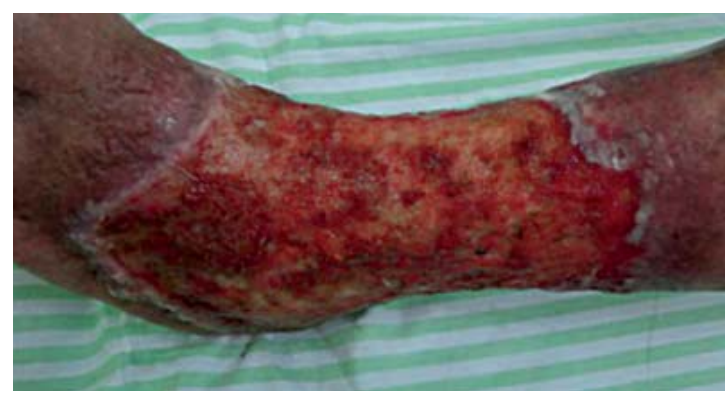

FIGURE 2: Lower left limb - May/2013

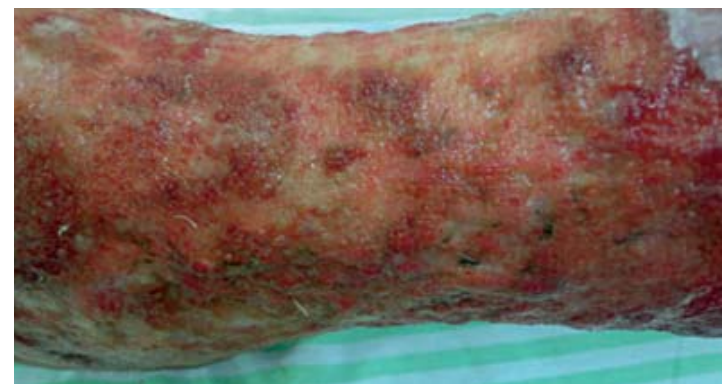

Figure 3: Detail of lower left limb - May/2013

\section{Obtaining the PRP}

Platelet-rich plasma is obtained through a process that utilizes the principle of cell separation by differential centrifugation. ${ }^{2}$ At present, there are at least 16 systems of PRP preparation available. ${ }^{3}$

At the outpatient clinic, at every application, $20 \mathrm{ml}$ of blood is collected and distributed into $5 \mathrm{ml}$ test tubes, containing sodium citrate solution $3.2 \%$, which are later centrifuged at $400 \mathrm{G}$ at $1500 \mathrm{RPM}$ for 15 minutes, at room temperature. Around $1.2 \mathrm{ml}$ of supernatant plasma is collected per tube and associated with 5 $\mathrm{ml}$ of calcium chloride at $0.025 \mathrm{~mol} / \mathrm{L}$. After approximately 5 minutes, the gel is formed (Figures 4 and 5). ${ }^{4}$ Later, it is applied to the wound bed and occluded with vaseline gauze and rolled bandage. The option of associating sodium calcium chloride and forming the platelet gel proved to be better than the intralesional PRP injection, since it is a large and painful ulcer.

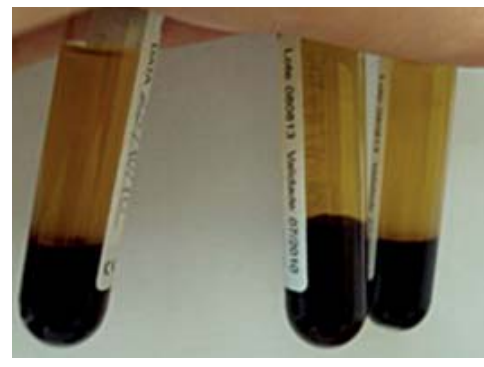

Figure 4:

Centrifuged blood

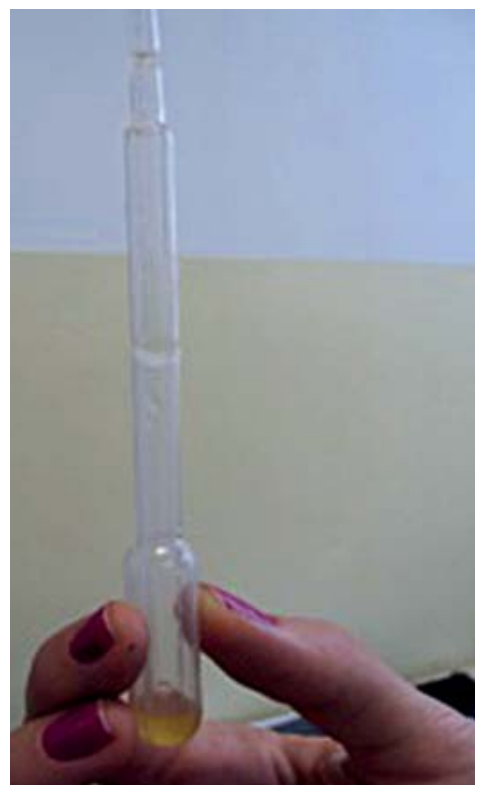

FiguRe 5:

Collected PRP

\section{DISCUSSION}

Due to life expectancy increase and, consequently, noticeable aging of the population, it is common for prevalence of chronic cutaneous ulcers to increase, notably when arising from atherosclerotic and microangiopathic processes. In this respect, it is necessary to develop techniques that assist in the process of skin healing and repair. PRP arrives as a tool which allows the application of large amounts of growth factors, which stimulate the production of collagen and extracellular matrix through minimum quantities of plasma. This is an important alternative in cases where conventional treatments were not successful, such as the one described.

Glycoprotein PDGF (platelet-derived growth factor) is the first growth factor to appear in the wound, starting the repair process of the connective tissue. Its most important specific activities include mitogenesis, angiogenesis, and macrophage activation. ${ }^{5}$

Growth factors promote quick increase of the number of undifferentiated mesenchymal cells at the scar site during repair and healing time. Therefore, the 
PRP advantage is to accelerate the regenerative process through the quantity of growth factors present in the platelets.

Amable et al conducted a study quantifying growth factors, cytokines and chemokines secreted by the platelet concentrate after calcium activation. High concentrations of PDGF, epidermal growth factor (EGF) and transforming growth factor (TGF) were secreted together with pro and anti-inflammatory cytokines like interleukin 4 (IL-4), IL-8, IL-13, IL-17, tumor necrosis factor alpha (TNF $\alpha)$ and interferonalpha (INF $\alpha)$. No cytokine was secreted before platelet activation. ${ }^{6}$

There are, however, many possible confounding variables in studies, as much regarding variation of patient characteristics as PRP characteristics. Thus the difficulty of conducting standardized studies. ${ }^{7}$ As regards research, it is a still recent discovery, although very promising.
The material is easily collected and processed, and the procedure of simple technical execution, with minimum morbidity, as it is totally autologous.

In spite of the advancements in cutaneous ulceration treatment, this common condition continues to devastate the community of patients who suffer from micro and macrovascular afflictions. The treatment with growth factors may result in shorter healing time and limb function recovery, enhancing the quality of life of the patient.

Based on literature, we conclude that the use of PRP in dermatology, albeit recent, constitutes a very promising technique. It is an organic preparation, non immunoreactive, non-toxic and of low morbidity. The cost of obtaining it is reasonably low.

Finally, more studies are necessary regarding the action mechanism and standardization of the ideal PRP preparation. Future studies should be conducted with lengthier follow-up periods and coverage of different PRP preparations.

\section{REFERENCES}

1. Crovetti G, Martinelli G, Issi M, Barone M, Guizzardi M, Campanati B, et al. Platelet gel for healing cutaneous chronic wounds. Transfus Apher Sci. 2004;30:145-51.

2. Mazzucco L, Medici D, Serra M, Panizza R, Rivara G, Orecchia S, et al. The use of autologous platelet gel to treat difficult-to-heal wounds: a pilot study. Transfusion. 2004; $44: 1013-8$

3. Saucedo JM, Yaffe MA, Berschback JC, Hsu WK, Kalainov DM. Platelet-Rich Plasma. J Hand Surg Am. 2012;37:587-9

4. Lenharo A, Cosso F. Fatores de crescimento: quando usar? Inovations J. 2001;5:21-5.

5. Marx RE, Carlson ER, Eichstaedt RM, Schimmele SR, Strauss JE, Georgeff KR. Platelet-rich plasma: growth factor enhancement for bone grafts. Oral Surg Oral Med Oral Pathol Oral Radiol Endod. 1998;85:638-46.

6. Amable PR, Carias RB, Teixeira MV, da Cruz Pacheco I, Corrêa do Amaral RJ, Granjeiro JM, et al. Platelet-rich plasma preparation for regenerative medicine: optimization and quantification of cytokines and growth factors. Stem Cell Res Ther. 2013;4:67.

7. Lacci KM, Dardik A. Platelet-rich Plasma: support for its use in wound healing. Yale J Biol Med. 2010;83:1-9.

\author{
MAILING ADDRESS: \\ Natássia Soares Pizani \\ Avenida Marques de Paraná, 303, térreo \\ Setor de Dermatologia \\ 24030-900 - Niterói - RJ \\ Brazil \\ E-mail:natassia.pizani@gmail.com
}

How to cite this article: Neffá Pinto JM, Pizani NS, Kang HC, Knecht Silva LA. Application of platelet-rich plasma in the treatment of chronic skin ulcer - Case report. An Bras Dermatol. 2014;89(4):638-40. 